\title{
The use of diagrams in analogical problem solving
}

\author{
ROBERTO PEDONE \\ University of Rome “La Sapienza," Rome, Italy \\ and \\ JOHN E. HUMMEL and KEITH J. HOLYOAK \\ University of California, Los Angeles, California
}

\begin{abstract}
In four experiments, we examined the impact of perceptual properties on the effectiveness of diagrams in analogical problem solving, using variants of convergence diagrams as source analogues for the radiation problem. Static diagrams representing the initial problematic state (one large line directed at a target) and the final state for a convergence solution (multiple converging lines) were not accessed spontaneously but were often used successfully once a hint to consider the diagram had been provided. The inaccessibility of static diagrams was not alleviated by adding additional diagrams to represent intermediate states (Experiment 1), but spontaneous access was improved by augmenting static diagrams with a verbal statement of the convergence principle (Experiment 3). Spontaneous retrieval and noticing were increased markedly by animating displays representing converging forces and thereby encouraging encoding of the lines as indicating motion toward a target (Experiments 3 and 4). However, neither static nor animated diagrams were effective when the arrows were reversed to imply divergence rather than convergence (Experiment 2). The results indicate that when animation encourages the interpretation of a diagram as a helpful source analogue, it can greatly enhance analogical transfer.
\end{abstract}

One of the most general methods for problem solving is to use a known source analogue as a guide to developing a solution for a novel target analogue. Many studies have demonstrated that both children and adults can solve problems by analogy (e.g., Gentner \& Gentner, 1983; Gick \& Holyoak, 1980, 1983; Holyoak, Junn, \& Billman, 1984; Holyoak \& Koh, 1987; Keane, 1986; Ross, 1987, 1989; for a review, see Holyoak \& Thagard, 1995, chap. 5). Virtually all of these studies have used verbal materials exclusively; relatively little work has explored the use of visual analogies. Most studies of diagrammatic reasoning have focused on the interpretation of pictures and diagrams that directly represent the information in the problem to be solved (e.g., by using a static picture of a pulley system to infer the direction of motion; see Ferguson \& Hegarty, 1995; Funt, 1995; Hegarty, 1991, 1992, 1995; Hegarty, Just, \& Morrison, 1988). In this line of research, the use of analogy between one problem and another has not been examined. In other work, the use of graphs to make inferences has been examined (Gattis \& Holyoak, 1996; Shah, Hegarty, \& Mayer, 1999). A graph (like a map) can be viewed as an analogue of what it represents but does not itself provide procedural information about how to solve a target problem. In contrast, pic-

This research was supported by NSF Grant SBR-9729023. We thank Steve Sloman and three anonymous reviewers for their helpful comments on earlier drafts. Correspondence should be addressed to J. E. Hummel, Department of Psychology, Franz Hall, UCLA, Los Angeles, CA 90095-1563 (e-mail: jhummel@ lifesci.ucla.edu). tures or diagrams that represent a distinct source analogue may enable the reasoner to construct a parallel procedure for solving the target problem.

A few experiments have shown that visual diagrams can be used as source analogues to solve a verbal target problem (Beveridge \& Parkins, 1987; Chen, 1995; Gick, 1985; Gick \& Holyoak, 1983). Gick and Holyoak (1983, Experiment 3) first showed college students the pair of diagrams depicted in Figure 1 and asked the participants to remember them so they could draw them later from memory. After a delay of a few minutes, the participants were given the task of solving the radiation problem (Duncker, 1945), in which a doctor must find a way to use a kind of ray to destroy an inoperable stomach tumor in a patient. The essence of the problem is that high-intensity rays would destroy the healthy tissue it passes through on the way to the tumor; although low-intensity rays would not harm the healthy tissue, they would be ineffective in treating the tumor.

The radiation problem thus has a rich semantic content, whereas the diagrams used by Gick and Holyoak (1983) were presented without any semantic interpretation. Nonetheless, it is possible to interpret the diagrams as a relational analogue to the radiation problem. Specifically, the left diagram could be interpreted as a large force directed at a target from a single point (analogous to the "obvious" but unsatisfactory idea of applying highintensity rays to the tumor), and the right diagram can be interpreted as representing a convergence solution, in which multiple small forces are directed from several 

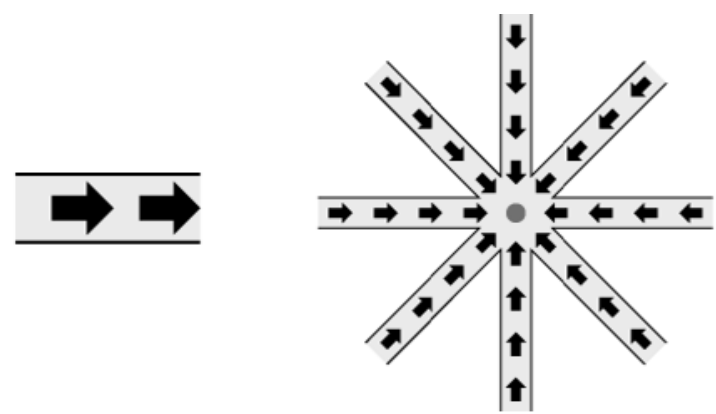

Figure 1. The pair of diagrams used as a source analogue by Gick and Holyoak (1983) and in Experiment 1 of the present study. From "Schema Induction and Analogical Transfer," by M. L. Gick and K. S. Holyoak, 1983, Cognitive Psychology, 15, p. 18. Copyright 1983 by Academic Press. Reprinted with permission.

points to converge at the target. If people can find the correspondences between the left diagram and the problem statement for the radiation problem, they might be able to use the right diagram to infer that an analogous convergence solution (simultaneous application of converging low-intensity rays to summate at the tumor) could be used to solve the radiation problem.

Successful use of diagrams as a source analogue, like use of a verbal source analogue, requires that the reasoner be able to carry out several mental processes. In particular, the reasoner needs to retrieve the source and notice its relevance when given the target problem, map the analogues to identify relational correspondences, and construct the analogous solution. Gick and Holyoak (1980, 1983) developed a paradigm for distinguishing the initial step of retrieval and noticing from the subsequent steps of mapping and solution generation. After presenting the source analogue in an incidental context, the participants were initially presented with the target problem without any hint that they should use the source analogue. If they were able to generate the analogous convergence solution at this point more often than participants in a control condition who had not received a source analogue, this would show they were able to both retrieve and use the source analogue. All the participants were then told that the source analogue might be helpful and were given a second opportunity to solve the target problem. If additional participants then gave the convergence solution, this would indicate they had initially failed to retrieve the source analogue but were nonetheless capable of performing mapping and solution generation.

One of the most general findings in Gick and Holyoak's $(1980,1983)$ experiments, for both verbal and diagrammatic source analogues, was that retrieval and noticing were major blocks to successful analogical problem solving. In the control condition (no source analogue), about $10 \%$ of the participants generated the convergence solution to the radiation problem. When the source was a verbal story highly dissimilar from the radiation problem (a story about a general using converging troops to capture a fortress), the rate of generating spontaneous convergence solutions was about $30 \%$. But after a hint to use the source was given, approximately an additional $50 \%$ of the participants gave the convergence solution, for a total solution rate of roughly $80 \%$. When the source analogue was the diagrams in Figure 1, the gap between prehint and posthint solution frequency was particularly extreme. Spontaneous (i.e., prehint) generation of the solution frequency did not differ from the baseline rate (i.e., $10 \%$ ), whereas the total solution rate once the hint was provided was about $70 \%$. Gick (1985) observed a similar gap between prehint and posthint solution rates with the diagrams as source. Similarly, research on diagrammatic reasoning has shown that diagrams are often most effective when accompanied by a text that explains how the diagrams should be interpreted (Hegarty \& Just, 1993; Mayer \& Gallini, 1990). An explanatory text accompanying a diagram thus seems to function much like a hint to use a diagram as a source analogue.

Why would spontaneous retrieval and noticing of a diagrammatic source analogue be so difficult? One general possibility is that without any semantic interpretation, the diagrams are not encoded in terms of concepts that could potentially link them to the verbal target problem. For example, the static line in the left diagram in Figure 1 need not be encoded as a representation of a direction of motion or of a force. It is, after all, simply a line with an arrow at one end. Once a hint to consider it has been given, the semantic interpretation of the target problem may be mapped "backward" onto the diagram, thereby bootstrapping the interpretation of the right diagram as the representation of the convergence solution, which can then be transferred to the radiation problem.

The question then arises of whether it is possible to use perceptual properties of diagrams to foster richer relational encodings, which would in turn make the diagrams more effective as source analogues, particularly by making spontaneous access easier. That is, without providing a verbal interpretation of the diagram, can its visuospatial properties suffice to make it an accessible source analogue? A study by Chen (1995) showed that transfer performance improves with increased similarity between a schematic source picture and a target problem; however, that study did not explore the possible use of more abstract diagrams. A study by Beveridge and Parkins (1987) provides suggestive evidence that diagrams can indeed be made more accessible. They used the same basic paradigm as Gick and Holyoak (1983) but varied the diagrams used as source analogues. The most effective analogue (Experiment 2) was a diagram in which the lines of force were represented by transparent blue plastic strips, hinged together at one end. As the participants watched, the strips were slowly fanned out to reveal the change from dark to light shades of blue on the nonoverlapping parts of the strip. When the colored strips were used as the source analogue, $95 \%$ of the participants spontaneously generated the convergence solution. Beveridge and Parkins 
suggested that, relative to the simpler diagrams used by Gick and Holyoak (1983), this visually presented "real" event provided a more explicit representation of the summative effect of intensity at the center of the display.

With the exception of the Beveridge and Parkins (1987) and Chen (1995) studies, there has been no systematic investigation of the impact of perceptual properties on the effectiveness of diagrams or pictures in analogical problem solving. In the present study, we report a series of experiments using variants of convergence diagrams as source analogues for the radiation problem. These variants differ in specific ways that might influence their relational encoding. In particular, we explored the impact of using longer sequences of diagrams that provided a denser representation of intervening problem states between the initial problematic state and the solution and the impact of introducing apparent motion to reinforce the interpretation of the lines as indicating directions of motion toward a target point. In line with Beveridge and Parkins's findings, we hypothesized that perceptual properties of diagrams that foster their interpretation as representations of forceful directed motion would facilitate their usefulness as source analogues ${ }^{1}$ for the radiation problem.

\section{EXPERIMENT 1}

The goal of Experiment 1 was to compare the usefulness of the pair of diagrams used by Gick and Holyoak (1983, Experiment 3) with that of a longer sequence of diagrams intended to depict intermediate states between the initial problematic state (one large force) and the convergence solution (several converging small forces).

\section{Method}

Participants. Forty-nine students in undergraduate psychology classes at the University of California, Los Angeles (UCLA) served in the experiment as part of a course requirement. Fifteen participants were assigned to the pictorial-diagram condition, 18 to the sequential-diagram condition, and 16 to the control condition.

Materials, Design, and Procedure. The basic procedure and materials were adapted from those employed by Gick and Holyoak (1983, Experiment 3). The three conditions differed in the source analogue provided. The participants assigned to the pictorial-diagram condition received the pair of diagrams depicted in Figure 1, which were identical to those used by Gick and Holyoak (1983, Experiment 3 ). Those assigned to the sequential-diagram condition received a sequence of diagrams, I through IV in the upper row of Figure 2. The participants in the control condition did not receive any diagrams or other source analogues. This control condition served to establish the base rate for production of convergence solutions in the absence of a source analogue.

The participants in the analogue conditions were told they would be serving in three different short experiments, the first on recall of pictures, the second on decision making, and the third on problem solving. As their initial task, the participants in the pictorial-diagram condition received the picture shown in Figure 1, displayed on a 15in. Macintosh computer screen. They were asked to study the diagram for $1 \mathrm{~min}$, after which they were asked to recall the diagram and write a verbal description of it from memory. The participants in the sequential-diagrams condition received the sequence of four convergence diagrams shown in the upper row of Figure 2. Dia- grams I and IV were identical to those shown together to the participants in the pictorial-diagrams condition. Diagrams II and III depicted intermediate states between a single large line and a single small line and a pair of converging lines, respectively. Each diagram in the sequence of four was displayed on the computer screen for $1 \mathrm{~min}$, after which the participants were asked to recall the diagrams and write a description of them.

After completing their initial task, all the participants completed an unrelated reasoning task that took about $5 \mathrm{~min}$. They then were presented a booklet that contained the radiation problem, stated as follows:

Suppose you are a doctor faced with a patient who has a malignant tumor in his stomach. It is impossible to operate on the patient, but unless the tumor is destroyed the patient will die. There is a kind of ray that can be used to destroy the tumor. If the rays reach the tumor all at once at a sufficiently high intensity, the tumor will be destroyed. Unfortunately, at this intensity the healthy tissue that the rays pass through on the way to the tumor will also be destroyed. At a lower intensity the rays are harmless to healthy tissue, but they will not affect the tumor either.

What type of procedure might be used to destroy the tumor with the rays, and at the same time avoid destroying the healthy tissue?

After they had read the problem, the participants were asked to produce as many solutions as possible. After $5 \mathrm{~min}$, the participants were told that the diagrams they had seen earlier might help them solve the problem. They were then asked to give whatever solution was suggested by the diagrams.

The participants in the control condition were simply given the radiation problem and asked to write down as many solutions to it as possible.

\section{Results and Discussion}

Solutions to the radiation problem were scored as to whether they explicitly or implicitly proposed the critical idea of having rays converge at the tumor. The basis for this assessment was the same as that used by Gick and Holyoak (1983). Solutions were judged by a scorer who was blind with respect to condition.

Table 1 presents the percentage of participants in each condition who produced the convergence solution to the radiation problem before the hint, after the hint, and in total. The percentage of participants producing the convergence solution to the target problem on their first pass (prehint) provides a measure of spontaneous noticing and application; the total percentage of participants producing the solution on either pass (prehint or posthint) provides a measure of potential application given that the analogy has been noticed. The difference between these two percentages provides a measure of the importance of having a "teacher" to point out the relevance of the prior source analogue.

The solution frequencies observed in the various conditions were compared using the maximum likelihood chisquare statistic, $G^{2}$ (Bishop, Fienberg, \& Holland, 1975). The results replicated and extended those obtained by Gick and Holyoak (1983). Prior to receiving a hint, the two analogue conditions did not differ from each other, nor did they yield more convergence solutions than did the control condition $\left[G^{2}(2)<1\right]$. The baseline solution frequency, approximately $13 \%$, was very similar to that obtained by Gick and Holyoak $(1980,1983)$. After the hint was provided, however, over $60 \%$ of the participants in 
CONVERGENCE

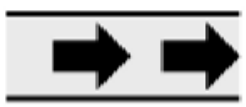

(I)

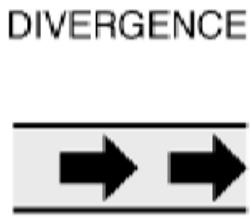

(I)

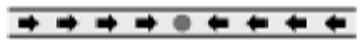

(II)

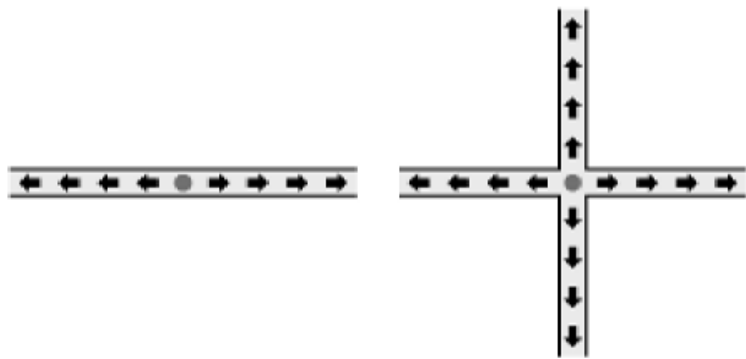

(II)

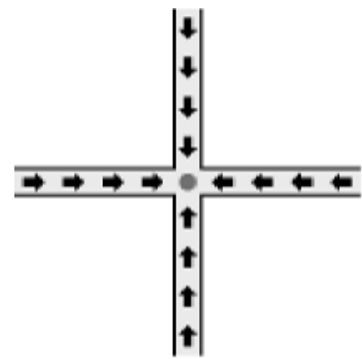

(III)

(III)

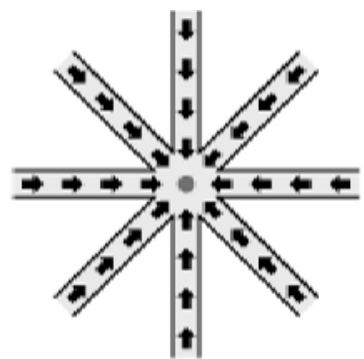

(IV)

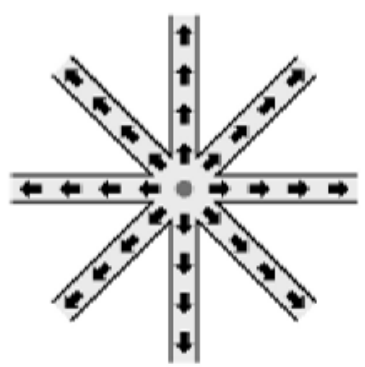

(IV)

Figure 2. Sequences of diagrams. Each of the four diagrams was presented in sequence, appearing alone on the screen. The diagrams in the upper row were used in the convergence conditions, and those in the lower row were used in the divergence conditions. Diagrams were not numbered in displays. Note that Diagram I was identical in the two sequences. In the static conditions, the diagrams appeared as they do in this figure. In the dynamic conditions, each diagram was animated, showing arrows in movement in the direction indicated by the arrows. Each dynamic diagram was composed of six different frames projected in a cycling fashion, with an interframe rate of $100 \mathrm{msec}$.

the two analogue conditions produced convergence solutions, greatly exceeding the performance of the control condition $\left[G^{2}(1)=12.42, p<.001\right]$. However, total solution frequencies did not differ across the two analogue conditions $\left[G^{2}(1)<1\right]$.

The results of Experiment 1 thus confirm Gick and Holyoak's (1983) finding that diagrams are not reliably accessed as source analogues without a hint but that they can be used effectively once their relevance is pointed out by a hint. Moreover, the present results show that the difficulty of spontaneously retrieving and noticing diagrammatic analogues is not reduced by presenting a sequence of diagrams to illustrate intermediate states. The computer-based display (sequential-diagrams condition) yielded a level of convergence solutions equivalent to that of the simpler pictorial-diagrams condition.

\section{EXPERIMENT 2}

The failure of the sequential-diagrams condition tested in Experiment 1 to increase solution transfer, relative to the pictorial-diagrams condition, indicates that neither use of a computer display nor depiction of intermediate states nor sequential presentation is sufficient to improve analogical transfer. However, Beveridge and Parkins (1987, Experiment 2) found a higher rate of transfer using a source analogue in which colored strips were physically manipulated to represent the effect of summation. One property of their display, which may have been critical, was that the analogue was, in effect, an animated display: The diagram underwent a continuous change (the colored strips were spread apart) as the participants watched it. Experiment 2 was designed specifically to examine the possible impact of animation on the effectiveness of diagrams as source analogues.

\section{METHOD}

Participants. Ninety-nine UCLA undergraduates participated in the experiment as part of a course requirement. They were assigned to one of five conditions, with 20 in each of four experimental conditions and 19 in the control condition.

Materials, Design, and Procedure. The basic procedure was the same as that used in Experiment 1. In four conditions, diagrammatic source analogues were used; in a control condition, the 
Table 1

Percentage of Participants Producing the Convergence Solution (Experiment 1)

\begin{tabular}{lcccc}
\hline \multicolumn{1}{c}{ Condition } & Before Hint & After Hint & Total & $N$ \\
\hline Control (no analogue) & - & - & 13 & 16 \\
Pictorial diagrams & 13 & 47 & 60 & 15 \\
Sequential diagrams & 17 & 50 & 67 & 18 \\
\hline
\end{tabular}

radiation problem was received without any analogue. Two of the analogues were based on the sequence of diagrams depicted in the top row of Figure 2. The participants in the static-convergence condition received the same sequential display of four diagrams as that in the sequential-diagrams condition of Experiment 1. Each diagram was displayed on a 15-in. Macintosh computer screen in turn for $1 \mathrm{~min}$, after which the participants were asked to recall the diagrams and write a verbal description of them from memory.

The participants in the dynamic-convergence condition saw an animated computer display based on the same four diagrams as those used in the static-convergence condition. Each diagram was displayed on the computer screen for $1 \mathrm{~min}$; each was animated, showing arrows in movement. Each diagram was composed of six different frames projected in a cycling fashion, with an interframe rate of $100 \mathrm{msec}$. The participants were then asked to recall the diagrams and write a verbal description of them from memory.

If the dynamic convergence displays yielded greater analogical transfer than did the static versions, one interpretation would be that any animated display improves transfer. To control for this possibility, the participants in two additional conditions were shown diagrams based on those depicted in the bottom row of Figure 2. These diagrams are matched to those in the top row of Figure 2, except that the arrows are directed outward instead of inward and hence represent divergence rather than convergence (Diagram I is identical in the two conditions). Since divergence is not a useful basis for solving the radiation problem, the divergence diagrams should be less effective than the convergence ones, even when animated. The static-divergence and dynamic-convergence conditions were respectively identical to their convergence counterparts, except for the change in the diagrams.

The same two-phase procedure was used as that in Experiment 1, with the same intervening filler task.

\section{Results and Discussion}

Convergence solutions were scored in the same manner as in Experiment 1. Table 2 presents the percentage of participants in each condition who produced the convergence solution to the radiation problem before the hint, after the hint, and in total. The baseline rate for the convergence solution in the control condition, which did not receive a source analogue, was $11 \%$. As in the identical condition in Experiment 1 (sequential diagrams), the prehint solution frequency for the static-convergence condition did not differ from the baseline $\left[G^{2}(1)<1\right]$. However, the rate of spontaneous transfer was much higher $(55 \%)$ for the dynamic-convergence condition, a rate reliably higher than that for the static-convergence condition $\left[G^{2}(1)=7.36, p<.01\right]$. The two convergence conditions did not differ significantly in total solution frequency $\left[G^{2}(1)=2.59, p=.10\right]$, but considered together, they yielded more total convergence solutions than did the control condition $\left[G^{2}(1)=27.59, p<.001\right]$.

The benefit of animation in spontaneous analogical transfer was restricted to the convergence conditions. The static-divergence and dynamic-divergence conditions did not differ in solution rates either prior to the hint $\left[G^{2}(1)=\right.$ $1.16, p>.20]$ or in total $\left[G^{2}(1)=1.60, p>.20\right]$; nor did the two divergence conditions considered together yield more convergence solutions than the control condition, either prior to the hint $\left[G^{2}(1)<1\right]$ or in total $\left[G^{2}(1)<1\right]$. The participants were thus extremely sensitive to the direction of motion indicated by the arrows in the diagram, so that only convergence diagrams, and especially the dynamic version, were effective source analogues for the radiation problem.

\section{EXPERIMENT 3}

Gick and Holyoak (1983) reported a number of findings indicating that analogical transfer is facilitated when the initial analogue (or multiple analogues) is encoded more abstractly. One way to potentially increase the abstraction of encoding is to augment the source analogue with an explicit verbal statement of the underlying solution principle. For diagrammatic source analogues, especially the static-convergence diagrams that are difficult to retrieve spontaneously, transfer may be facilitated by adding a statement of the principle.

\section{Method}

Participants. Forty UCLA undergraduates served in the experiment as part of a course requirement, with 20 assigned to each of the two conditions.

Materials, Design, and Procedure. Two conditions were tested, the static-convergence and the dynamic-convergence conditions. The materials, design, and procedure were identical to those used for these two conditions in Experiment 2, except that during the time the diagrams were displayed, the following sentence was shown at the bottom of the computer screen: "If you need a large force to accomplish some purpose, but are prevented from applying such force directly, many smaller forces applied simultaneously from different directions may work just as well." This verbal principle had been used previously by Gick and Holyoak (1983).

\section{Results and Discussion}

Table 3 presents the percentage of participants in each condition who produced the convergence solution to the radiation problem before the hint, after the hint, and in total. With the addition of the verbal principle, analogical transfer was quite robust in both conditions. Prior to the hint, about $50 \%$ of the participants in both the staticconvergence and the dynamic-convergence conditions succeeded in producing the convergence solution, with no significant difference between the two groups $\left[G^{2}(1)<\right.$

Table 2

Percentage of Participants Producing the Convergence Solution (Experiment 2)

\begin{tabular}{lcccc}
\hline \multicolumn{1}{c}{ Condition } & Before Hint & After Hint & Total & $N$ \\
\hline Control (no analogue) & - & - & 11 & 19 \\
Static convergence & 15 & 55 & 70 & 20 \\
Dynamic convergence & 55 & 35 & 90 & 20 \\
Static divergence & 15 & 10 & 25 & 20 \\
Dynamic divergence & 5 & 5 & 10 & 20 \\
\hline
\end{tabular}


Table 3

Percentage of Participants Producing Convergence Solution (Experiment 3)

\begin{tabular}{lcccc}
\hline Condition & Before Hint & After Hint & Total & $N$ \\
\hline Static convergence + principle & 45 & 25 & 70 & 20 \\
Dynamic convergence + principle & 50 & 45 & 95 & 20 \\
\hline
\end{tabular}

1]. The static-convergence condition with the principle tested in Experiment 3 produced a higher frequency of prehint solutions than did the comparable static-convergence condition without a principle, tested in Experiment 2 $\left[G^{2}(1)=4.44, p<.05\right]$. Total solution rate after the hint in Experiment 3 was greater in the dynamic than in the static condition [ $95 \%$ vs. $\left.70 \% ; G^{2}(1)=4.72, p<.05\right]$. Thus, the addition of a verbal principle facilitated spontaneous retrieval of the source analogue in the staticconvergence condition but did not entirely eliminate the overall advantage of the dynamic condition.

\section{EXPERIMENT 4}

Both Experiments 2 and 3 provided evidence that the dynamic-convergence diagrams are more effective as source analogues than are their static counterparts. However, it would be desirable to test whether the advantage of dynamic displays extends to other variants of the diagrams. The results of Experiment 2 indicated that dynamic displays are not effective when the arrows are reversed, thereby indicating divergence rather than convergence. In Experiment 4, we examined another modification of the diagrams, in which the arrows were replaced by blocks. Although blocks do not convey direction of motion as clearly as do arrows, they are compatible with a convergence interpretation (unlike the reversed arrows used in the divergence conditions of Experiment 2). Since a dynamic display provides additional cues to motion, we predicted that diagrams with blocks, instead of arrows, would yield greater transfer when the displays were dynamic rather than static.

\section{Method}

Participants. Forty UCLA undergraduates served in the experiment as part of a course requirement, with 20 assigned to each of the two conditions.

Materials, Design, and Procedure. Two conditions were tested, the static-convergence and the dynamic-convergence conditions. The materials, design, and procedure were identical to those used for these two conditions in Experiment 2, except that the diagrams used were based on those depicted in Figure 3. The sole change between these diagrams and those used previously (upper row in Figure 2) is that the arrows have been replaced by undirected blocks.

\section{Results and Discussion}

Table 4 presents the percentage of participants in each condition who produced the convergence solution to the radiation problem before the hint, after the hint, and in total. The dynamic-convergence condition tended to produce a higher rate of transfer both prior to the hint $\left[G^{2}(1)=\right.$ $4.72, p<.05]$ and in total $\left[G^{2}(1)=5.01, p<.05\right]$. The results of Experiment 4 thus indicate that dynamic displays yield more robust transfer than do static displays for multiple variants of the diagrams, as long as they can be interpreted as representing convergence.

\section{GENERAL DISCUSSION}

The results of the present study provide a broad picture of the impact of various perceptual properties of diagrams on their effectiveness as source analogues for solving the verbal convergence problem. Static diagrams representing the initial problematic state (one large line directed at a target) and the final state for a convergence solution (multiple converging lines) are not accessed spontaneously but can often be used successfully, once a hint to consider the diagram has been provided. The inaccessibility of sta-
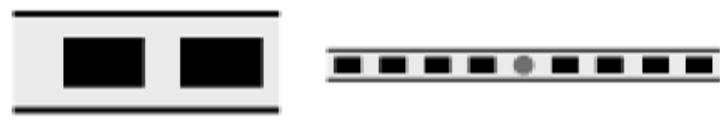

(l)
(II)

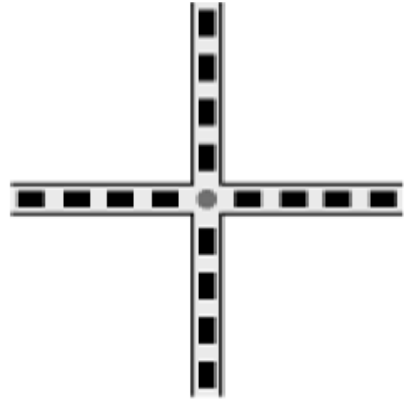

(III)

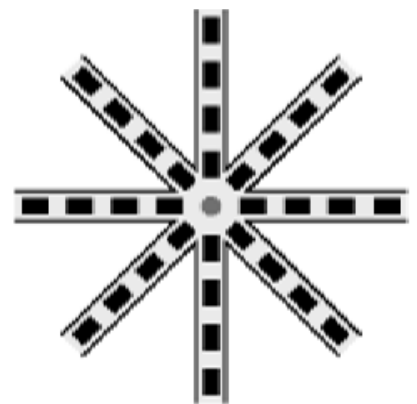

(IV)

Figure 3. Sequence of diagrams used in Experiment 4. Each of the four diagrams was presented in sequence, appearing alone on the screen. In the static condition, the diagrams appeared as they do in this figure. In the dynamic condition, each diagram was animated, showing blocks in movement. Each dynamic diagram was composed of six different frames projected in a cycling fashion, with an interframe rate of $100 \mathrm{msec}$. 
Table 4

Percentage of Participants Producing Convergence Solution (Experiment 4)

\begin{tabular}{lcccc}
\hline \multicolumn{1}{c}{ Condition } & Before Hint & After Hint & Total & $N$ \\
\hline Static convergence & 5 & 30 & 35 & 20 \\
Dynamic convergence & 30 & 40 & 70 & 20 \\
\hline
\end{tabular}

tic diagrams is not alleviated by adding additional diagrams to represent intermediate states (Experiment 1), but spontaneous access can be improved by augmenting static diagrams with a verbal statement of the convergence principle (Experiment 3 ).

The most important result, however, is that spontaneous retrieval and noticing can be markedly improved by animating the display in a manner that encourages encoding of the lines as indicating motion toward a target. The advantage of dynamic over static diagrams for representing convergence was obtained both when the diagrams included directed arrows (Experiment 2) and when they instead included undirected blocks (Experiment 4). However, neither static nor animated diagrams were effective when the arrows were reversed to imply divergence rather than convergence (Experiment 2). Although representing only a small change perceptually, the reversed arrows radically alter the interpretation of the direction of motion in a way that makes it extremely difficult for reasoners to map the diagrams onto the radiation problem and infer a convergence solution. Thus, animation is helpful only when it encourages an interpretation of the diagram that, in fact, can be mapped onto the target problem.

The effectiveness of animated convergence in making diagrams function effectively as source analogues for the convergence solution to the radiation problem is consistent with the findings of Beveridge and Parkins (1987), who showed that enacting summation with colored strips also led to a high rate of analogical transfer. The effectiveness of dynamic versions of convergence diagrams is particularly striking in that people often accessed the diagrams spontaneously despite the complete absence of a verbal "cover story" to guide their interpretation. In Experiment 2, over half the participants in the dynamicconvergence condition produced the convergence solution to the radiation problem prior to a hint. This high rate of spontaneous transfer is substantially greater than that typically observed with verbal far analogues (e.g., the fortress analogue used by Gick \& Holyoak, 1980, 1983; Spencer \& Weisberg, 1986). In other words, whereas static diagrams representing convergence yield minimal spontaneous transfer (less than verbal far analogues), dynamic versions of the same displays are actually better than verbal source analogues that are semantically rich but superficially dissimilar.

There are multiple possible explanations for the potency of dynamic displays of convergence as source analogues. It may be that with the aid of apparent motion, people are led to encode the displays as something like abstract schemas for convergence. Appropriate animation may thus function much like the provision of multiple analogues (Catrambone \& Holyoak, 1989; Gick \& Holyoak, 1983 ) or the addition of an abstract verbal principle (Gick \& Holyoak, 1983; present Experiment 3). Another possibility (not mutually exclusive with the first) is that dynamic displays result in encodings that are more closely connected to the perceptual system than can be readily achieved by purely verbal materials. That is, actually seeing a display in motion may yield a representation of moving forces that is more purely perceptual than the kind of representation generated by reading text. The convergence solution depends, in a deep sense, on understanding the perceptual and physical reality of how converging forces interact with each other and with other objects that they contact along their paths. This type of understanding may be best conveyed by animated displays that generate rich perceptual representations.

The present findings were obtained solely with diagrammatic analogues to the radiation problem. Further research will be required to examine the usefulness of other types of diagrams in solving other types of problems and also to determine the exact mechanisms underlying the effectiveness of dynamic displays. If it can be shown that appropriate animation of the source analogue facilitates analogical transfer to other problems, then animationeven, and perhaps particularly, animation of abstract diagrams - may prove to be a practical tool for education.

\section{REFERENCES}

Beveridge, M., \& Parkins, E. (1987). Visual representation in analogical problem solving. Memory \& Cognition, 15, 230-237.

Bishop, Y. M. M., Fienberg, S. E., \& Holland, P. W. (1975). Discrete multivariate analysis: Theory and practice. Cambridge, MA: MIT Press.

Catrambone, R. \& Holyoak, K. J. (1989). Overcoming contextual limitations on problem-solving transfer. Journal of Experimental Psychology: Learning, Memory, \& Cognition, 15, 1147-1156.

Chen, Z. (1995). Analogical transfer: From schematic pictures to problem solving. Memory \& Cognition, 23, 255-269.

DUNCKER, K. (1945). On problem solving. Psychological Monographs, 58 (Whole No. 270).

Ferguson, E. L., \& Hegarty, M. (1995). Learning with real machines or diagrams: Application of knowledge to real-world problems. Cognition \& Instruction, 13, 129-160.

Funt, B. V. (1995). Problem solving with diagrammatic representations. In J. Glasgow, N. H. Narayanan, \& B. Chandrasekaran (Eds.), Diagrammatic reasoning: Cognitive and computationalperspectives (pp. 33-68). Cambridge, MA: MIT Press.

Gattis, M., \& Holyoak, K. J. (1996). Mapping conceptual to spatial relations in visual reasoning. Journal of Experimental Psychology: Learning, Memory, \& Cognition, 22, 231-239.

Gentner, D., \& Gentner, D. R. (1983). Flowing waters or teeming crowds: Mental models of electricity. In D. Gentner \& A. L. Stevens (Eds.), Mental models (pp. 99-129). Hillsdale, NJ: Erlbaum.

GICK, M. L. (1985). The effect of diagram retrieval cue on spontaneous analogical transfer. Canadian Journal of Psychology, 39, 460-466.

Gick, M. L., \& Holyoak, K. J. (1980). Analogical problem solving. Cognitive Psychology, 12, 306-355.

Gick, M. L., \& Holyoak, K. J. (1983). Schema induction and analogical transfer. Cognitive Psychology, 15, 1-28.

Hegarty, M. (1991). Knowledge and processes in mechanical problem 
solving. In R. J. Sternberg \& P. A. Frensch (Eds.), Complex problem solving: Principles and mechanisms (pp. 253-285). Hillsdale, NJ: Erlbaum.

Hegarty, M. (1992). Mental animation: Inferring motion from static displays of mechanical systems. Journal of Experimental Psychology: Learning, Memory, \& Cognition, 18, 1084-1102.

Hegarty, M. (1995). Mental animation. In J. Glasgow, N. H. Narayanan, $\&$ B. Chandrasekaran (Eds), Diagrammatic reasoning: Cognitive and computational perspectives (pp. 535-576). Cambridge, MA: MIT Press.

Hegarty, M., \& Just, M. A. (1993). Constructing mental models of machines from text and diagrams. Journal of Memory \& Language, 32, 717-742.

Hegarty, M., Just, M. A., \& Morrison, I. R. (1988). Mental models of mechanical systems: Individual differences in qualitative and quantitative reasoning. Cognitive Psychology, 20, 191-236.

Holyoak, K. J., JunN, E. N., \& Billman, D. (1984). Development of analogical problem-solving skill. Child Development, 55, 2042-2055.

HolyoAK, K. J., \& KoH, K. (1987). Surface and structural similarity in analogical transfer. Memory \& Cognition, 15, 332-340.

Holyoak, K. J., \& Thagard, P. (1995). Mental leaps: Analogy in creative thought. Cambridge, MA: MIT Press.

Hummel, J. E., \& HolyoaK, K. J. (1997). Distributed representations of structure: A theory of analogical access and mapping. Psychological Review, 104, 427-466.

KeANE, M. T. (1986). On retrieving analogues when solving problems. Quarterly Journal of Experimental Psychology, 39A, 29-41.

MAYER, R. E., \& Gallini, J. K. (1990). When is an illustration worth ten thousand words? Journal of EducationalPsychology, 82, 715-726.
Ross, B. (1987). This is like that: The use of earlier problems and the separation of similarity effects. Journal of Experimental Psychology: Learning, Memory, \& Cognition, 13, 629-639.

Ross, B. (1989). Distinguishing types of superficial similarities: Different effects on the access and use of earlier problems. Journal of Experimental Psychology: Learning, Memory, \& Cognition, 15, 456468.

Shah, P., Hegarty, M., \& Mayer, R. E. (1999). Graphs as aids to knowledge construction: Signaling techniques for guiding the process of graph comprehension. Journal of Educational Psychology, 91, 690-702.

SPEnCER, R. M., \& WeISBERG, R. W. (1986). Context-dependenteffects on analogical transfer. Memory \& Cognition, 14, 442-449.

\section{NOTE}

1. Insofar as diagrams can be interpreted as representations of the abstract convergence principle, they could be construed as schemas, rather than as specific analogues (Gick \& Holyoak, 1983). However, theories of analogy generally consider the process of mapping to be performed by fundamentally the same mechanism regardless of whether the source is an abstract schema or a specific analogue (e.g., Hummel \& Holyoak, 1997). For this reason and because a diagram is inherently a specific visual object, we will refer to the diagrams used in this study as source analogues.

(Manuscript received July 28, 1999; revision accepted for publication August 3, 2000.) 